\title{
Practical Tips to Ensure Patient Safety while Managing Cases of Placenta Praevia
}

ISSN: 2640-9666

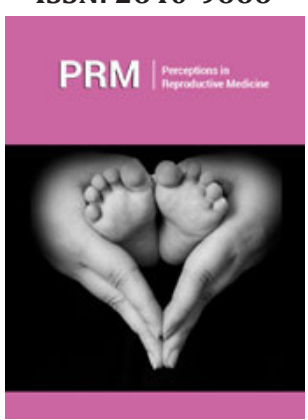

*Corresponding author: Vidyadhar Bangal, Department of Obstetrics and Gynecology, India

Submission: 漈 October 30, 2020

Published: 佂 November 06, 2020

Volume 4 - Issue 2

How to cite this article: Vidyadhar Bangal. Practical Tips to Ensure Patient Safety while Managing Cases of Placenta Praevia. Perception in Reproductive Medicine. 4(2). PRM. 000583. 2020. DOI: 10.31031/PRM.2020.04.000583

Copyright@ Vidyadhar Bangal, This article is distributed under the terms of the Creative Commons Attribution 4.0 International License, which permits unrestricted use and redistribution provided that the original author and source are credited.

\author{
Vidyadhar Bangal* \\ Department of Obstetrics and Gynecology, India
}

\section{Background}

On many occasions Obstetrician encounter problems or difficulties while managing cases of placenta praevia or while performing caesarean section for placenta praevia. At times, patients loose large amount of blood leading to shock. Excessive and unanticipated blood loss pose the risk of severe maternal morbidity or mortality.

Following are practical tips for managing cases of placenta praevia.

\section{A-Antenatal Woman Diagnosed to Have Placenta Praevia, who is not Bleeding}

a. Admit the patient and follow standard protocol of conservative management in antenatal ward.

b. Arrange minimum two units of blood. Ask relatives to donate blood.

c. Do not perform per vaginal and frequent per abdominal examination.

d. Let everyone including nursing staff know about the admitted case, so that anyone can start the zero-hour management, in case patient starts bleeding at any odd hour.

e. Do not change or shift the bed of the patient. Keep the bed close to passage for prompt transfer on stretcher.

f. Allow one female relative with the patient all the time. Write emergency contact number of the patient's first degree relative on the case sheet front page back side.

g. Take provisional consent for Obstetric hysterectomy, internal iliac ligation, massive blood transfusion and admission to intensive care unit before shifting patient to operation theatre. Additional consents may be obtained during surgery as and when it is needed depending upon intra-operative findings and some unanticipated complication.

h. Plan termination of pregnancy by appropriate method at the completion of $36 / 37$ weeks. There is no advantage of conservative management after completed 37 weeks.

i. Rule out placental invasion by ultra-sonography by senior sonologists. Resident must accompany the patient when patient is shifted for sonography or to operation theatre on stretcher.

j. Reconfirm the blood and component availability before posting the case for surgery

k. Confirm the availability of responsible relative of the patient at the time of surgery.

l. Post the placenta praevia caesarean section during routine morning OT, when whole team is available for help. Considering the unanticipated intra operative problems, never post elective placenta praevia caesarean section at night time.

m. Arrange/confirm the availability of two faculty members/obstetrician during surgery. 


\section{B-Antenatal Woman Diagnosed to have Placenta Praevia, who is Bleeding}

a. Secure two IV line, use No 16/18 vein flow, collect adequate cross matching sample, start Ringers Lactate iv solution.

b. Arrange two units of PCV and four FFP.

c. Arrange/confirm the availability of two faculty members/ Obstetrician during surgery.

d. Mobilize adequate manpower(Residents and Interns).

e. Shift patient to operation theatre after taking high risk consent.

f. After opening the abdomen, inspect for evidence of abnormal vascularity of the uterus, especially lower uterine segment. See if there is any evidence of increta and percreta, bladder involvement, prominent vessels in broad ligament.

g. Do not be in hurry to give incision on lower segment without proper inspection of the uterus. Do not blindly believe USG findings regarding invasion. Call for help when needed before opening the uterus.

h. Perform surgery/caesarean as per clinical and ultrasonographic evaluation of the case.

i. If there is evidence of increta or percreta, perform classical caesarean section avoiding extension of vertical incision in lower uterine segment, deliver the baby, do not disturb the placenta, apply temporary haemostatic clamps to both cornua and isthmus. Plan internal iliac artery ligation followed by obstetric hysterectomy. j. Call surgeon, if bladder invasion is anticipated or seen on inspection before opening the uterus.

k. Inform anesthesiologists about the surgical plan or anticipated blood loss and operation time so that they make necessary arrangement at their end.

l. If there is un-anticipated bleeding from lower uterine segment, then ask second assistant to apply good aortic compression, so as to control placental site bleeding till further preparations/planning for surgery is done. Apply good aortic compression using fist of the hand, so that there is disappearance of femoral pulsation.

m. Do not fiddle with the placenta, if you feel that it is adherent. Attempts of manual separation of adherent placenta can result into profuse torrential bleeding resulting into hypovolemic shock and even cardiac arrest within very short time.

n. If placenta is seen posterior and adherent, inspect the posterior surface of the uterus externally by exteriorization. Judge the extent of invasion and plan further actions accordingly.

It is extremely important to anticipate the complications and thus make necessary arrangements before posting the cases (elective as well as emergency) of placenta praevia for surgery.

Be prepared to deal with adherent placenta or placental site bleeding. Make sure that the operating surgeon/surgeons are well versed with the procedures like internal iliac ligation, aortic compression and obstetric hysterectomy. 